\title{
Heterotopic Pancreas of the Jejunum Incidentally Detected by Preoperative Abdominal CT: Report of Two Cases and Review of the Literature
}

\author{
Manabu Watanabe ${ }^{a}$ Kazue Shiozawa $^{a}$ Yui Kishimoto ${ }^{a}$ \\ Tsunetaka Arai $^{\mathrm{a}}$ Shigeru Nakano ${ }^{\mathrm{a}}$ Yoshinori Kikuchi $^{\mathrm{a}}$ \\ Takashi Ikehara $^{a}$ Yoshinori Igarashi ${ }^{a}$ Daisuke Sasai $^{b}$ \\ Yasukiyo Sumino ${ }^{a}$ \\ ${ }^{a}$ Division of Gastroenterology and Hepatology, Department of Internal \\ Medicine, and ${ }^{\mathrm{b}}$ Department of Surgical Pathology, Toho University Medical \\ Center, Omori Hospital, Tokyo, Japan
}

\section{Key Words}

Heterotopic pancreas - Computed tomography - Small bowel submucosal tumor . Incidentaloma $\cdot$ Capsule endoscopy

\begin{abstract}
Heterotopic pancreas (HP) is typically an asymptomatic malformation that can present anywhere along the gastrointestinal tract. It is often detected incidentally on surgery for other diseases or autopsy. We encountered 2 patients with jejunal HP incidentally detected by computed tomography (CT) performed for close evaluation of other diseases. In a 57-year-old woman diagnosed with reactive lymphoid hyperplasia on the dorsal portion of the pancreas head, CT detected a $15 \mathrm{~mm}$ oval-shaped submucosal lesion at the jejunum. In an 87-year-old woman diagnosed with type 2 adenocarcinoma occupying the sigmoid colon, CT detected a round-shaped submucosal tumor $15 \mathrm{~mm}$ in diameter in the jejunum. Both cases were histologically diagnosed as type $1 \mathrm{HP}$ according to the classification by Heinrich. Contrast-enhanced $\mathrm{CT}$ revealed that the $\mathrm{CT}$ analyses of HP and pancreatic parenchyma were nearly identical in the arterial phase, but in the equilibrium phase, contrast enhancement persisted longer in HP than in the pancreatic parenchyma. There has been no report of asymptomatic jejunal HP preoperatively diagnosed by $\mathrm{CT}$. These cases are presented with a review of the literature, particularly focusing on CT findings.
\end{abstract}




\section{Introduction}

Heterotopic pancreas (HP) is pancreatic tissue that develops in areas other than the normal site of the pancreas, having no anatomical or vascular continuity with the normal pancreas [1, 2]. HP of the small intestine, if enlarged, may be diagnosed due to symptoms such as ileus, bowel intussusception, melena and pancreatitis, but it often remains asymptomatic and is incidentally detected on surgery for other diseases or autopsy [3, 4]. We encountered 2 patients with jejunal HP incidentally detected by computed tomography (CT) performed for close evaluation of other diseases. There has been no report of asymptomatic jejunal HP preoperatively diagnosed by CT. These cases are presented with a review of the literature, particularly focusing on CT findings.

\section{Case Reports}

Case 1 was a 57-year-old woman who had been under antihypertensive medication for a few years. When she consulted a local physician due to epigastric and back pain, a tumoral lesion $30 \mathrm{~mm}$ in diameter was detected on the dorsal portion of the pancreas head on abdominal ultrasonography, and the patient was referred to our hospital. No abnormality was noted on blood chemistry tests or tumor marker measurements. The mass was suspected to be schwannoma, Castleman disease, malignant lymphoma or reactive lymphoid hyperplasia by imaging studies including CT and magnetic resonance imaging. CT also revealed a mass $15 \mathrm{~mm}$ in diameter in the jejunum. The contrast of the mass was nearly homogeneously enhanced in the arterial phase, and enhancement was sustained in the equilibrium phase, suggesting a submucosal tumor ( $\underline{\mathrm{fig}} \mathbf{1} \mathrm{a}, \mathrm{b}$ ). Double contrast intestinal imaging showed a defect of about $14 \mathrm{~mm}$ in diameter in the jejunum, and a gently elevated submucosal mass with a smooth mucosal surface was confirmed by subsequent capsule endoscopy (fig. 1c) and small intestinal endoscopy. Diseases including gastrointestinal stromal tumor and leiomyoma were suspected preoperatively, and laparotomy was performed to simultaneously remove the masses in the head of the pancreas and small intestine. The mass in the head of the pancreas was intraoperatively suspected to be disadherable, with enlarged \#8p and \#13a lymph nodes, and was eventually diagnosed by histopathological examination as reactive lymphoid hyperplasia. Also, a tumor $15 \mathrm{~mm}$ in diameter was noted in the jejunum $15 \mathrm{~cm}$ anally from the ligament of Treitz, and an about $10 \mathrm{~cm}$ segment of the jejunum was resected with the tumor. It was histopathologically diagnosed as Heinrich type $1 \mathrm{HP}$ mainly occupying the submucosa to the muscular layer (fig. 1d)

Case 2 was an 87-year-old woman who presented with fecal occult blood on a health screening and was referred to our hospital. Blood tests indicated anemia with a $\mathrm{Hb}$ level of $9.1 \mathrm{~g} / \mathrm{dl}$ (normal 12.0-16.0). As for tumor makers, CEA was elevated to $8.4 \mathrm{ng} / \mathrm{ml}$ (normal 0-5.0) and p53 antibody to $3.44 \mathrm{ng} / \mathrm{ml}$ (normal $0-5.0$ ). Colonic fiberscopy showed type 2 adenocarcinoma occupying two thirds of the circumference of the sigmoid colon. CT disclosed a mass about $15 \mathrm{~mm}$ in diameter in the jejunum in addition to a sigmoid colon tumor accompanied by swollen lymph nodes in the neighboring regions. The image of the jejunal mass was homogeneously contrasted in the atrial phase, and contrast enhancement persisted in the equilibrium phase, suggesting a submucosal tumor (fig. 2). Double contrast intestinal imaging showed a gently elevated semispherical mass $14 \mathrm{~mm}$ in diameter with a smooth mucosal surface near the ligament of Treitz. Surgery was performed under laparoscopic guidance to resect the sigmoid colon cancer and jejunal submucosal tumor. After sigmoid colectomy (double stapling technique, D3), a tumor $15 \mathrm{~mm}$ in diameter was found in the jejunum $5 \mathrm{~cm}$ anally from the ligament of Treitz and was removed by wedge resection. It was diagnosed histopathologically to be Heinrich type 1 HP seated mainly in the submucosa.

CT images were obtained by using multi-detector row CT (MDCT) scanner (LightSpeed VCT, GE Healthcare) in both patients. Both patients received the contrast agent Moiopamin 300 (dose $100 \mathrm{ml}$ ) through the median cubital vein. The arterial and equilibrium phases were approximately 35 and $120 \mathrm{~s}$, respectively, after the start of injection. Retrospectively, the CT values of the HP tissue and pancreatic parenchyma were calculated in both phases. In the region of interest in the pancreatic parenchyma, CT values were measured at three points in the body of the pancreas by avoiding 
pancreatic ducts and vessels, and the mean was calculated. Concerning HP, the region of interest was determined around the mass in the axial plane that provided the largest image of the mass on CT. The CT values in cases 1 and 2 are shown (fig. 3). In case 1, the CT values in HP and the pancreatic parenchyma were 106 and $100 \mathrm{HU}$, respectively, in the arterial phase and 101 and 75 HU, respectively, in the equilibrium phase. In case 2, the CT values in HP and the pancreatic parenchyma were 124 and $122 \mathrm{HU}$, respectively, in the arterial phase and 91 and $77 \mathrm{HU}$, respectively, in the equilibrium phase. The CT values of HP and pancreatic parenchyma were nearly identical in the arterial phase, but in the equilibrium phase, contrast enhancement persisted longer in HP than in the pancreatic parenchyma.

\section{Discussion}

HP lacks both anatomical and vascular continuity with the main body of the pancreas and occurs anywhere from the esophagus to the rectum [1, 2]. The etiology of HP is unclear, although various theories have been put forward. HP is often detected incidentally or on autopsy. The incidence of HP varies widely and has been reported in $0.6-5.6 \%$ of autopsy cases and in $0.2 \%$ of upper abdominal laparotomies [1]. The distribution of the sites of HP was $30.3 \%$ in the duodenum, $26.5 \%$ in the stomach, $16.3 \%$ in the jejunum, 5.8\% in the ileum and 5.3\% in Meckel diverticulum, indicating that it occurs more frequently near the normal site of pancreatogenesis to the jejunum. These lesions are usually clinically silent but may become symptomatic because of complications such as pancreatitis, obstruction, bleeding or malignant transformation $[3,4]$. A review of the literature reveals that the significance of HP symptoms is related to the size and mucosal relation of the lesion. In a review of 34 histologically confirmed cases, these authors concluded that lesions associated with signs and symptoms are $>1.5 \mathrm{~cm}$ in maximum diameter and are adjacent to or directly involve the mucosa [5]. A definitive HP diagnosis may be established on the basis of histological examination, which additionally allows confirmation of the histological type of HP. Heinrich's classification is commonly accepted, being as follows: type 1 (all elements of a normal pancreatic gland), type 2 (pancreatic gland devoid of pancreatic islet cells), and type 3 (only pancreatic ducts are present). Both of the present cases were type 1 according to Heinrich [6].

The preoperative diagnosis of HP in the small bowel appears to be difficult. Also, there had been no report of delineation of asymptomatic and small jejunal $\mathrm{HP} \leq 1.5 \mathrm{~cm}$ in diameter by preoperative CT before our cases. On the other hand, CT findings of HP in the stomach and duodenum have recently been reported [7-10]. CT findings of HP in the stomach or duodenum are usually nonspecific and cannot distinguish HP from other submucosal tumors. However, CT with arterial, portal and equilibrium phase IV contrast may demonstrate the lesions which enhance similarly with the normal pancreatic tissue [7]. Kim et al. [8] reported that HP with predominantly pancreatic acini shows a homogeneous enhancement pattern, whereas lesions with a mixed composition of acini and cystic ducts show a heterogeneous enhancement. The lesions in both of our patients corresponded to Heinrich type 1 with no ductal dilation or fat deposition, and showed homogeneous enhancement similarly to the pancreatic parenchyma in the arterial and equilibrium phases on CT. However, the CT value of the jejunal HP was nearly identical with that of the pancreatic parenchyma in the arterial phase, but was higher in the equilibrium phase in both patients. The cause of the difference in the CT value in the equilibrium phase is unknown, but it may reflect not only the difference in the composition of HP tissue but also atrophy, fibrosis and fat 
degeneration of the pancreatic parenchyma. As gastric or duodenal HP is difficult to differentially diagnose from other submucosal tumors such as leiomyoma, carcinoid and gastrointestinal stromal tumor by the contrast enhancement pattern on CT or morphology alone, HP of the small intestine is difficult to differentiate from other diseases by CT alone. The detection rate of HP of the small intestine is expected to increase with the future spread of MDCT. With increasing case numbers, contrast patterns in particular phases will be evaluated under appropriate conditions, and MDCT may contribute to the differential diagnosis of HP of the small intestine.

There is no consensus about whether incidentally detected asymptomatic HP should be resected. Particularly, when it is detected intraoperatively, the judgment is difficult as there is no preoperative informed consent for its resection. Since the possibility of its canceration is extremely low and as it grows very slowly, preventive resection has been reported to be unnecessary $[11,12]$. Recently, however, there have been a number of reports of its resection due to melena, ileus, intussusception, etc. even without canceration, and many authors support aggressive resection of resectable HP. In our cases, jejunal HP was detected incidentally during examination for other diseases, and the presence of submucosal masses could be confirmed preoperatively by fluoroscopy and endoscopy of the small intestine. This made it possible to obtain sufficient informed consent from the patients to remove the intestinal submucosal tumor as well as the primary disease and to perform surgery safely.

This report presented 2 cases of jejunal HP incidentally detected by CT during examination for other diseases. The frequency of incidental detection of intestinal submucosal tumors is expected to increase with a rise in the frequency of MDCT use. While most small lesions of HP are asymptomatic, it may be complicated by ileus and intussusception, and HP is considered to be an important disorder that should be recognized along with gastrointestinal stromal tumor, carcinoid, etc. 

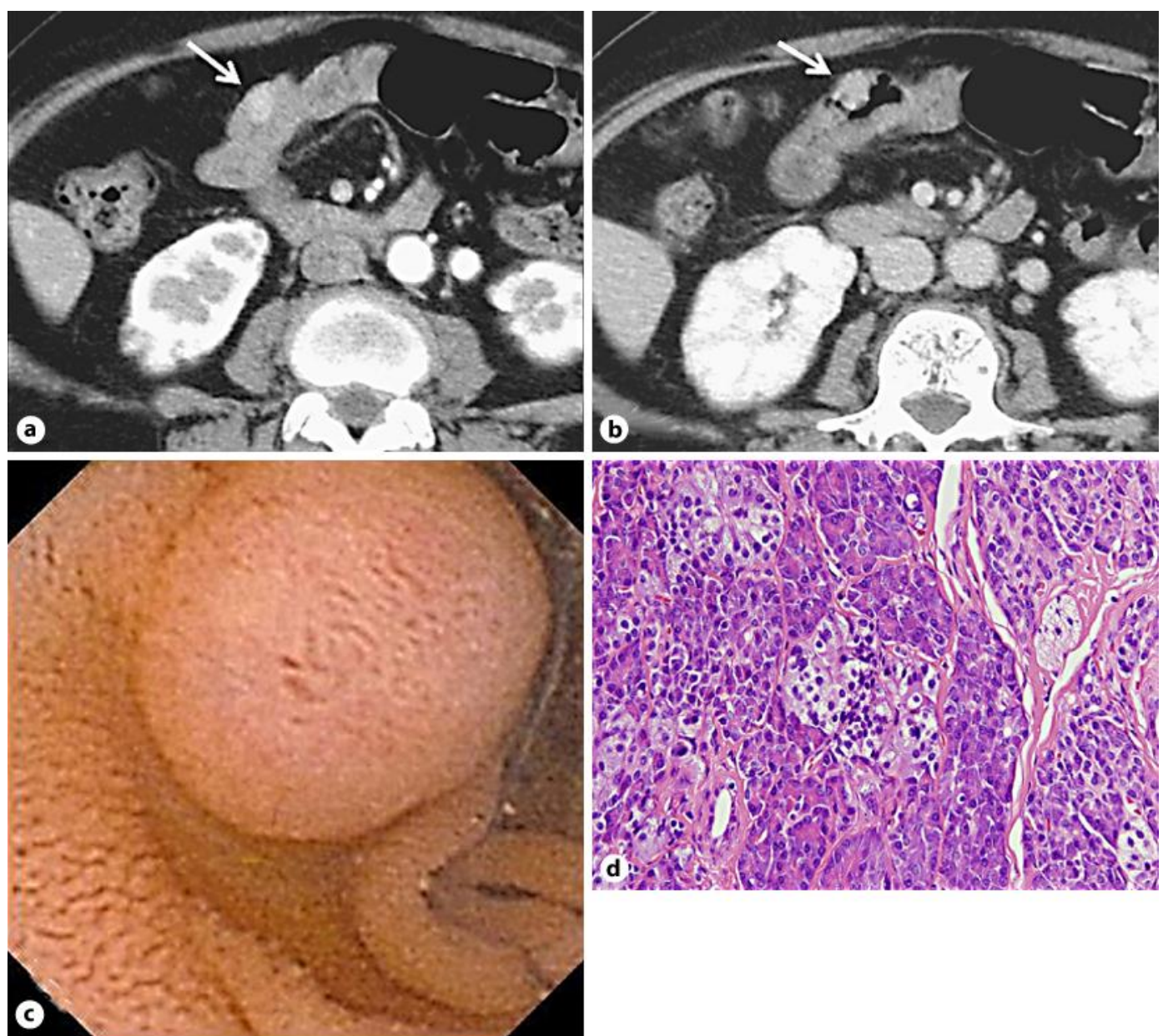

Fig. 1. Contrast-enhanced CT showed a $15 \mathrm{~mm}$ well-demarcated oval submucosal lesion at the jejunum. The lesion (arrow) showed homogenous and strong enhancement in the arterial phase (a), and enhancement was sustained in the equilibrium phase (b). c Capsule endoscopy showed a gently elevated submucosal mass with a smooth mucosal surface in the jejunum. $\mathbf{d}$ Histological findings showed pancreatic tissue composed of pancreatic acini, pancreatic ducts and islets of Langerhans. Hematoxylin-eosin stain, original magnification $\times 40$. 


\begin{tabular}{r|l|l|l}
$\begin{array}{r}\text { Case Reports in } \\
\text { Gastroenterology }\end{array}$ & $\begin{array}{l}\text { Case Rep Gastroenterol 2012;6:576-582 } \\
\text { DOI: 10.1159/000343093 }\end{array}$ & $\begin{array}{l}\text { Published online: } \\
\text { September 11, 2012 }\end{array}$ & $\begin{array}{l}\text { @ 2012 S. Karger AG, Basel } \\
\text { ISSN 1662-0631 } \\
\text { www.karger.com/crg }\end{array}$ \\
\hline
\end{tabular}
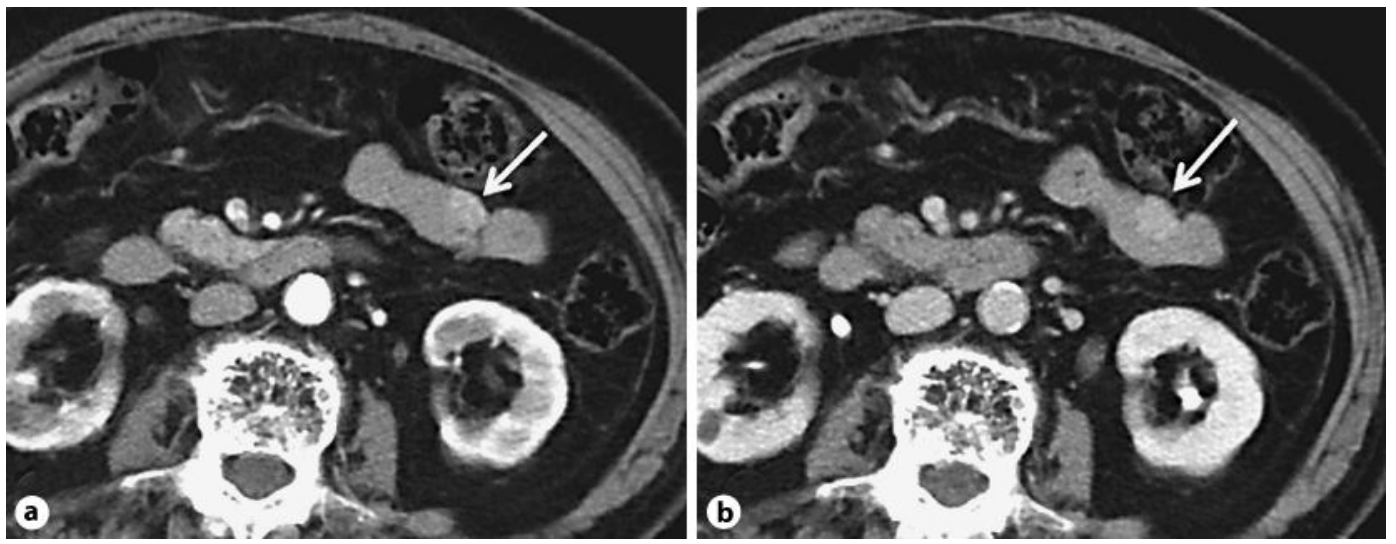

Fig. 2. Contrast-enhanced CT showed a $15 \mathrm{~mm}$ oval submucosal lesion at the jejunum. The jejunal mass (arrow) was homogeneously contrasted in the atrial phase (a), and contrast enhancement persisted in the equilibrium phase (b).
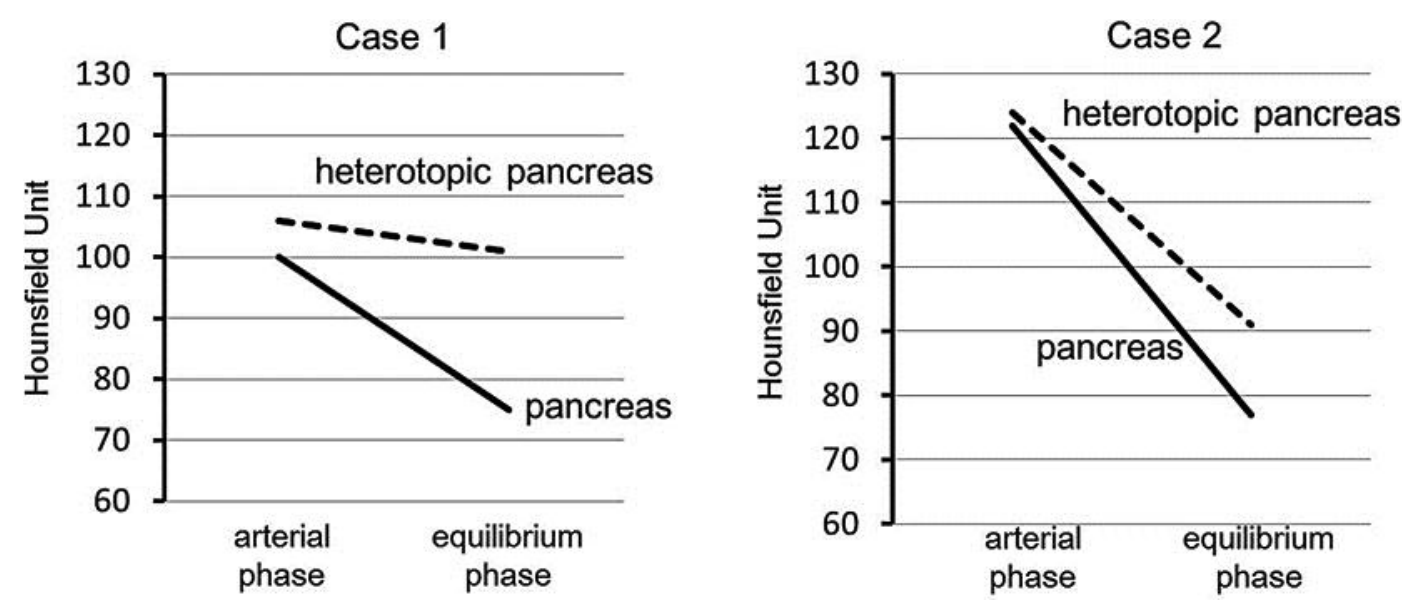

Fig. 3. The CT values in cases 1 and 2 are shown. In case 1 , the CT values in HP and the pancreatic parenchyma were 106 and $100 \mathrm{HU}$, respectively, in the arterial phase and 101 and $75 \mathrm{HU}$, respectively, in the equilibrium phase. In case 2 , the CT values in HP and the pancreatic parenchyma were 124 and $122 \mathrm{HU}$, respectively, in the arterial phase and 91 and $77 \mathrm{HU}$, respectively, in the equilibrium phase.

\section{References}

1 Barbosa JJ, Docketry MB, Waugh JM: Pancreatic heterotopia; review of the literature and report of 41 authenticated surgical cases, of which 25 were clinically significant. Surg Gynecol Obstet 1946;82: 527-542.

2 Dolan RV, ReMine WH, Dockerty MB: The fate of heterotopic pancreatic tissue: a study of 212 cases. Arch Surg 1974;109:762-765.

3 Christodoulidis G, Zacharoulis D, Barbanis S, Katsogridakis E, Hatzitheofilou K: Heterotopic pancreas in the stomach: a case report and literature review. World J Gastroenterol 2007;13:6098-6100.

4 Eisenberger CF, Gocht A, Knoefel WT, Busch CB, Peiper M, Kutup A, Yekebas EF, Hosch SB, Lambrecht W, Izbicki JR: Heterotopic pancreas - clinical presentation and pathology with review of the literature. Hepatogastroenterology 2004;51:854-858. 
5 Armstrong CP, King PM, Dixon JM, Macleod IB: The clinical significance of heterotopic pancreas in the gastrointestinal tract. Br J Surg 1981;68:384-387.

6 von Heinrich H: Ein Beitrag zur Histologie des sogen. akzessorischen Pankreas. Virchows Arch A Pathol Anat Histopathol 1909;198:392-401.

-7 Cho JS, Shin KS, Kwon ST, Kim JW, Song CJ, Noh SM, Kang DY, Kim HY, Kang HK: Heterotopic pancreas in the stomach: CT findings. Radiology 2000;217:139-144.

-8 Kim JY, Lee JM, Kim KW, Park HS, Choi JY, Kim SH, Kim MA, Lee JY, Han JK, Choi BI: Ectopic pancreas: CT findings with emphasis on differentiation from small gastrointestinal stromal tumor and leiomyoma. Radiology 2009;252:92-100.

9 Wang D, Wei XE, Yan L, Zhang YZ, Li WB: Enhanced CT and CT virtual endoscopy in diagnosis of heterotopic pancreas. World J Gastroenterol 2011;17:3850-3855.

10 Park SH, Han JK, Choi BI, Kim M, Kim YI, Yeon KM, Han MC: Heterotopic pancreas of the stomach: CT findings correlated with pathologic findings in six patients. Abdom Imaging 2000;25:119-123.

11 Persson GE, Boiesen PT: Cancer of aberrant pancreas in jejunum. Case report. Acta Chir Scand 1988;154:599-601.

12 Feldman M, Weinberg T: Aberrant pancreas: a cause of duodenal syndrome. JAMA 1952;148:893-898. 